\title{
Towards Possible Cure of Cancer by Immunotherapy of Minimal Residual Disease
}

\author{
Shimon Slavin ${ }^{1 *}$, Nadir Askenasy ${ }^{1}$ and Chaya Brodie ${ }^{2,3}$ \\ ${ }^{1}$ International Center for Cell Therapy and Cancer Immunotherapy (CTCI), Tel Aviv 64239, Israel \\ ${ }^{2}$ Mina and Everard Goodman Faculty of Life-Sciences, Bar-Ilan University, Ramat-Gan, Israel \\ ${ }^{3}$ Hermelin Brain Tumor Center, Department of Neurosurgery, Henry Ford Hospital, Detroit, MI, USA
}

*Corresponding author: Shimon Slavin, International Center for Cell Therapy and Cancer Immunotherapy (CTCI), Tel Aviv 64239, Israel, Tel: $972-77$ 777 9255; E-mail: slavinMD@gmail.com

Rec date: Sep 22, 2014, Acc date: April 27, 2015, Pub date: May 7, 2015

Copyright: () 2015 Slavin S, et al. This is an open-access article distributed under the terms of the Creative Commons Attribution License, which permits unrestricted use, distribution, and reproduction in any medium, provided the original author and source are credited.

\begin{abstract}
Based on predictive, preventive and personalized medicine (PPPM) strategy for treatment of cancer, immunotherapy and additional targeted anti-cancer modalities should be considered for cure of cancer at an early stage of the disease following successful conventional treatment. Alternatively, PPPM strategy should be applied at the stage of minimal residual disease (MRD) induced following successful re-induction of second or subsequent remission.

Fortunately, a stage of minimal residual disease can be accomplished in most patients with cancer following conventional treatment but in the absence of visible disease or lack of symptoms, additional treatment is usually denied. Considering PPPM using innovative and safe treatment focusing on immunotherapy should be considered for patients at risk because eradication of MRD can be accomplished whereas treatment of overt disease may no longer be possible. Similarly to the treatment of infectious diseases, the goal should be to recognize the high-risk cases upfront and treat MRD because residual malignant cells cannot be detected by available imaging technologies.
\end{abstract}

The take home message is that at the stage of minimal residual disease focusing on immunotherapy and other targeted anti-cancer modalities may be safe, successful and possibly the only way to cure cancer, or at least to better control tumor progression.

Keywords: Cancer immunotherapy; Minimal residual disease (MRD); Personalized medicine; Alloreactive lymphocytes (T Cells And NK Cells); Cell-mediated anti-cancer immunotherapy

\section{Introduction}

Cancer continues to be among the leading causes of death worldwide. Considering lack of information about the causes of malignant transformation, except when viral agents seem to be involved, the universal dogma for treatment of cancer is based on 'the more the better' using available anti-cancer agents, attempting to eradicate the primary tumor by resection with or without chemotherapy or radiation therapy when indicated as well as the secondary metastases as soon as they become visible with aggressive chemotherapy as the standard of care. For patients with cancer not expected to respond adequately to conventional treatment myeloablative chemoradiotherapy followed by rescue with stem cell transplantation may provide an alternative opportunity for eradication of maximal possible number of tumor cells. Over the years it became apparent that in patients with primary resistant cancer or with metastatic disease none of the available anti-cancer modalities or even combinations thereof could accomplish/eradication of all residual malignant cells, since relapse continues to be the single major obstacle in treatment of hematologic malignancies and many solid tumors as well even following initial complete remission $[1,2]$. On the other hand, as the intensity of the regimen used for treatment of cancer or using high dose chemotherapy supported by stem cell transplantation (SCT) is escalated, the risks of procedure-related toxicity and mortality increase accordingly [3].

In addition, increased incidence and severity of late complications of patients treated with higher or repeated doses of chemotherapy became an important issue too in evaluating the quality of life of longterm survivors. It became apparent that newer modalities must be introduced in order to improve the cure rate of patients with/ hematologic malignancies as well as to improve the quality of life of successfully treated patients. For patients resistant to available chemotherapy, immunotherapy became an obvious rational alternative. Unfortunately, in the absence of tumor-specific antigens the value of immunotherapy based on tumor cell vaccines is questionable [4]. Clearly, the goal of effective immunotherapy should be based on an attempt to induce an 'autoimmune-like' response against tumor cells recognized as 'self [5]. To date, effective tumorassociated antibodies, such as anti-CD20 in patients with B cell nonHodgkin's lymphoma or Herceptin against her-2/neu that can effectively control tumor progression in responding patients, are not sufficient for eradication of the disease [6].

Considering the fact that cancer cells consistently acquire resistance to chemotherapy and other available anti-cancer modalities, and that cancer stem cells are a priori resistant to available anti-cancer agents, 
new approaches are urgently indicated for treatment of otherwise incurable cancer. Newer treatment strategies are needed in an attempt to control invisible residual malignant cells resistant to chemotherapy, which at the stage of MRD may not be detectable. It should be remembered that every cancer begins with a single cancer initiating cell and since even one million cancer cells are not visible by available imaging technologies that cannot detect one millimeter size lesion, there is an indication to treat invisible MRD in high risk patients that are likely to retain resistant cancer cells following conventional treatment although residual disease is not visible. Besides, cancer stem cells are a priori resistant to available modalities. Therefore, treatment of MRD by a safe immunotherapy program that can eliminate cancer cells despite resistance to chemotherapy and radiation seems to be one of the most promising future approaches for improving the treatment of cancer.

As will be suggested in this short chapter, immunotherapy and additional targeted anti-cancer modalities applied at the stage of MRD which can be easily accomplished in a large number of patients at an early stage of the disease may be the optimal approach for cure of cancer. Aiming for cure or long-term disease-free or progression-free survival, such treatment should be best applied at an early stage of the disease, following successful application of conventional treatment that may consist of surgery in the case of solid tumors, with additional first line chemotherapy if indicated, or following successful chemotherapy combinations or chemo-radiotherapy in the case of malignant hematological diseases.

Considering the need for innovative future approaches for treatment of cancer, it seems that predictive, preventive and personalized medicine (PPPM), should be best applied for treatment of cancer, simply because all available modalities for treatment of advanced cancer are far from being satisfactory. Considering the fact that there are no two cancers that are exactly the same and no two patients that are exactly the same, future progress for the treatment of cancer will be based on early treatment of the malignant process at the stage of minimal residual disease, on a fully personalized basis, again, based on the principles of PPPM.

\section{The role of immunotherapy by alloreactive lymphocytes}

Following successful animal experiments, the clinical role of allogeneic lymphocytes was introduced in patients with leukemia successfully treated by allogeneic bone marrow transplantation (BMT). The role of allogeneic BMT, explored originally by Thomas et al. in the early 1970s became the treatment of choice for/ patients resistant to conventional doses of chemotherapy, and subsequently for patients at high-risk to relapse following maximally tolerated doses of conventional chemotherapy [7]. Subsequently, BMT was successfully utilized for the treatment of genetic diseases and other life-threatening non-malignant indications using the same therapeutic principles for replacement of abnormal host hematopoietic cells with donor hematopoietic cells.

Traditionally, it was considered that high-dose chemoradiotherapy was the main component in the bone marrow transplant procedure and that transplantation of genotypically or phenotypically matched stem cells was mainly indicated for rescue of the lethally treated recipient. Hence, much attention was given to maximize tumor cell kill by maximally tolerated doses of chemotherapy (single agents and combinations of non-cross reactive agents).
However, it was recognized for many years that the/incidence of relapse was higher among recipients of autologous as well as syngeneic grafts as compared with recipients of allogeneic grafts with graftversus-host disease (GVHD), suggesting that immune-mediated graftversus-leukemia $(\mathrm{GVL})$ effects played a major role in elimination of residual tumor cells escaping chemoradiotherapy [8-11].

The possibility that allogeneic lymphocytes administered in the course of BMT eliminate leukemia through immune-mediated GVL effects has been suggested ever since the earliest days of experimental [12-19] and clinical BMT [8-11]. Convincing direct correlation between acute and chronic GVHD and reduced rate of relapse of leukemia in clinical practice was first reported by Weiden et al. [8,9].

Similarly, in analogy to GVL effects graft-versus-tumor (GVT) effects were also described in a murine model of spontaneous sarcoma [20] and more recently in metastatic breast cancer as well $[21,22]$, as well as in preliminary trials in man [23-25]. The role of immunemediated GVL effects in the course of BMT was further supported by observations suggesting that relapse while patients were on immunosuppressive treatment with preliminary trials in man [23-25].

The role of immune-mediated GVL effects in the course of BMT was further supported by observations suggesting that relapse while patients were on immunosuppressive treatment with cyclosporine A was occasionally reversed by discontinuing immunosuppression [26]. Likewise, it has been documented that the incidence of relapse is lower in patients treated with sub optimal doses of CSA [27]. In support, data in mice inoculated with murine leukemia treated by BMT indicated that GVL effects mediated by mismatched bone marrow cells were totally abrogated by concomitant administration of CSA for 10 days [28].

All of the above suggest that allogeneic BMT provided immunocompetent allogeneic donor $\mathrm{T}$ lymphocytes, which could react against residual tumor cells of host origin. Hence, the advantage of BMT over conventional chemotherapy lies in the combined effects of the myeloablative dose of chemoradiotherapy given pretransplantation and the ability of immunocompetent allogeneic donor $\mathrm{T}$ lymphocytes to eliminate residual tumor cells of host origin, giving rise to GVL and GVT effects or in fact graft versus any undesirable hematopoietic cells of host origin, including genetically abnormal stem cells or their progeny [29-32].

Interestingly, similarly to the data first reported in mice [14-18], GVL effects independently of GVHD were also confirmed in clinical practice either following BMT [11] or following donor lymphocyte infusion (DLI) given post transplantation to induce GVL effects to treat or prevent relapse when patients are off any post-transplant immunosuppressive agents [33-40].

Based on the pre-clinical animal model data that suggested the feasibility of induction of post-transplant GVL effects induced by T cells present in the allografts, we hypothesized that cell-therapy with donor lymphocytes given post grafting, especially in patients with no spontaneous GVHD following discontinuation of post-transplant antiGVHD prophylaxis, may induce effective anti-tumor responses [5].

\section{The use of durable engraftment of alloreactive donor lymphocytes following allogeneic SCT for immunotherapy of cancer}

Based on the beneficial role of allogeneic stem cell transplantation in comparison with autologous stem cell transplantation and especially 
transplantation of patients following equal high dose chemotherapy supported by stem cell transplantation obtained from identical twins, we hypothesized that allogeneic lymphocytes of donor origin can be given post grafting for treatment as well as for prevention of relapse in high-risk cases. Indeed, the first successful case where GVL effects were induced by allogeneic cell-therapy, the so-called DLI, in a patient with resistant acute lymphoblastic leukemia (ALL) with fully resistant disease, followed by hundreds of patients successfully treated in Israel, subsequently supported by the cumulative international experience in a variety of malignant hematologic diseases confirmed unequivocally the therapeutic potential of alloreactive lymphocytes [33-40].

The first patient successfully treated by DLI for relapse following BMT was a 30 month old boy that was referred for BMT at the Hadassah University Hospital /in Jerusalem in November 1986 [33-35]. He had been diagnosed as pre-B ALL and relapsed on therapy twice. In December 1986, allogeneic BMT was carried out from a fully matched sister during second resistant relapse.

Supra-lethal conditioning included total body irradiation (TBI) $1,200 \mathrm{cGy}$ (two daily fractions of $200 \mathrm{cGy}$ on days $-6,-5$ and -4 ) followed by two doses of cyclophosphamide $60 \mathrm{mg} / \mathrm{kg}$ (days -3 and -2) and melphalan $60 \mathrm{mg} / \mathrm{m} 2$ (day -1). The patient showed no signs of acute GVHD. At one-month post-BMT, the patient presented with full hematologic relapse and several bulky masses confirmed as extramedullary disease, including a progressing retro-tracheal mass necessitating an emergency tracheotomy.

He responded to increments of donor (sister) peripheral blood lymphocytes infusions to induce GVL effects. The patient developed grade II GVHD with involvement of the skin and liver. He responded to a short course of corticosteroids and within 2 weeks the palpable masses decreased in size, peripheral blood and bone marrow morphology normalized and cytogenetic analysis confirmed 100\% normal female karyotype in all 50 metaphases investigated.

To date, more than 25 years after treatment with DLI following his third relapse post BMT, no residual male cells are detected by PCR analysis of either Y-specific or amelogenin gene markers. The efficacy of DLI for eradication of malignant cells fully resistant to lethal doses of chemo-radiotherapy was confirmed by many centers [36-40] and currently, DLI is considered the treatment of choice for patients relapsing following allogeneic stem cell transplantation [41].

Considering the anti-cancer potential of alloreactive lymphocytes, the use of pre-emptive DLI was introduced by our team for prevention of relapse following BMT [42]. The therapeutic role of DLI could be further maximized by in vitro or in vivo activation of alloreactivity of lymphocytes by interleukin-2 [35]. In later years, the well-documented role of alloreactive donor lymphocytes lead to the replacement of myeloablative conditioning for allogeneic BMT with reduced intensity conditioning (RIC) and non-myeloablative stem cell transplantation (NST) $[43,44]$ as documented diagrammatically in Figure 1. Subsequently, the role of reduced intensity conditioning (RIC) as a means to induce host-vs.-graft unresponsiveness that allows durable engraftment of alloreactive donor lymphocytes was also pioneered for the treatment of solid tumors [45-47].

\section{Replacing myeloablative with reduced intensity conditioning} The rationale for a safer conditioning strategy

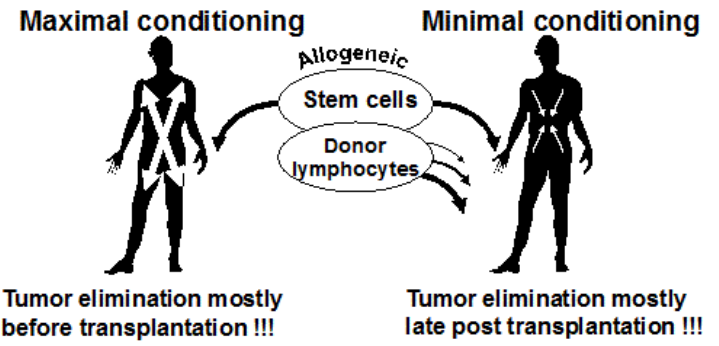

Figure 1: Diagrammatic documentation of the difference between the "classical" approach for allogeneic stem cell transplantation focusing on myeloablative conditioning and reduced intensity conditioning (RIC) or non-myeloablative stem cell transplantation (NST). Myeloablative conditioning was originally designed for trying to eradicate all malignant cells by maximally tolerated doses of chemo-radiotherapy, using the stem cell transplantation for rescue of lethally conditioned recipient. In sharp contrast, RIC or NST were designed for induction of host-vs.-graft unresponsiveness by engraftment of donor hematopoietic stem cells that allows durable engraftment of donor lymphocytes including $\mathrm{T}$ cells and NK cells for induction of graft-versusleukemia or graft-vs.-tumor effects. GVL or GVT effects could be amplified by post-transplant administration of donor lymphocytes, the so-called donor lymphocyte infusion (DLI) for prevention or treatment of recurrent disease.

\section{The therapeutic role of transient circulation of intentionally mismatched alloreactive killer cells (IMAK)}

The therapeutic role of allogeneic lymphocytes intentionally mismatched and IL-2 activated for rapid elimination of malignant cells without the use of SCT for induction of host-versus-graft unresponsiveness was pioneered in a 12 years old patient with AML resistant to high dose chemotherapy supported by autologous stem cell transplantation in 1992 [48]. A stage of MRD was induced in a patient with AML with evidence of disease following induction chemotherapy by myeloablative chemotherapy and hematopoietic reconstitution with autologous stem cells. Minimal residual disease was successfully eliminated by haploidentically mismatched maternal lymphocytes activated with IL-2 in vitro prior to cell infusion and subsequently by administration of IL-2 to allow continuous activation of alloreactive donor lymphocytes until the anticipated rejection of killer T cells and natural killer cells [48]. Currently, more than 20 years later, the patient, a qualified MD with 2 children, is alive and well, free of any signs of disease. This patient was the first one to confirm that even transient engraftment of alloreactive lymphocytes could eliminate malignant cells fully resistant to myeloablative chemotherapy. 
The benefit of alloreactive donor lymphocytes for eradication of chemotherapy and radiation resistant disease was also documented in a patient with non-Hodgkin's lymphoma [49].

More recently, the use of intentionally mismatched killer cells was pioneered in patients with metastatic solid tumors and also in patients with hematological malignancies. Following successful animal experiments, we have documented that transient engraftment of alloreactive lymphocytes with minimal conditioning with a single low dose cyclophosphamide to allow for homeostatic expansion of donor lymphocytes and also for down regulation of regulatory $\mathrm{T}$ cells can be effective against malignant cells resistant to conventional anti-cancer modalities [50]. Our cumulative experience, which needs to be extended in prospective randomized clinical trials, suggests that at the stage of MRD cure of patients with hematologic malignancies and metastatic solid tumors otherwise considered incurable, may benefit or even cured by cellular therapy [50]. Interestingly, the therapeutic role of non-alloreactive haploidentically mismatched cells following allogeneic SCT and depletion of immunocompetent $\mathrm{T}$ cells described by Ruggeri et al. [51] and Velardi et al. [52] also confirms the potential beneficial anti-cancer effects of durably engrafting mismatched donor cells independently of GVHD. In contrast, the anti-cancer effects induced by IMAK are mediated by both IL- 2 activated alloreactive T cells and NK cells induced by non-engrafting donor lymphocytes. Since donor lymphocytes are expected to be rejected after no longer than one week, it is essential that aiming for cure, treatment should be applied at the stage of MRD.

The selectivity and efficacy of intentionally mismatched killer cells can be improved by targeting of lymphocytes to malignant cells by monoclonal or bispecific antibodies [53]. Moreover, treatment of MRD with antibody targeted killer cells may also result in induction of long-lasting anti-cancer immunity, most likely because the Fc portion of the monoclonal or bispecific antibody can bind to antigen presenting cells (dendritic cells or macrophages) leading to processing of cancer antigens and presentation to helper $\mathrm{T}$ cells [54].

\section{Immunotherapy of MRD by activation of host immune system}

Immunotherapy of cancer with monoclonal or bispecific antibodies may be an effective way to induce antibody-dependent cell-mediated cytotoxicity which can be accomplished by using commercially available antibodies such as anti-CD20 (MabThera), anti her-2/neu (Herceptin), anti-EGFR (Erbitux), anti-VEGF (Avastin) and bispecific antibodies such as anti-CD3xanti-EpCAM (Catumaxomab) that are entering clinical practice.

Whereas the use of transient circulation of alloreactive donor lymphocytes makes it possible to eliminate truly MRD, in principle, successful activation of patient's own immune system against cancer makes it possible to induce long-lasting immune reactivity against residual malignant cells. Methods to stimulate patients' own immune system against cancer include non-specific activation of $\mathrm{T}$ cells and NK cells with IL-2 alone or combination of IL-2 and interferon. We have previously documented that such treatment may be effective for treatment of leukemia/lymphoma in pre-clinical animal experiments [5] and in pilot clinical trials $[55,56]$. Treatment with IL-2 and alpha interferon significantly improved the disease-free survival and overall survival of patients with Hodgkin's and non-Hodgkin's lymphoma treated with high dose chemotherapy and autologous stem cell transplantation prior to cytokine treatment $[57,58]$. Anti-cancer effects were also reported following treatment with macrophage activating factor (GcMAF) [59,60].

The use of anti-cancer vaccination by loading tumor cell lysate or tumor-associated peptides on dendritic cells seems to be other methods for attempting to induce anti-cancer effects.

Induction of anti-cancer effects may be improved by using concomitant metronomic treatment against negative regulators of the immune system such as regulatory T cells or prostaglandin E2.

\section{Other methods for treatment of residual disease in patients with cancer}

Control of cancer cells by low molecular compounds like tyrosine kinase inhibitors represent one of the truly breakthrough treatment of cancer initially documented in patients with Philadelphia-positive CML [61]. Similar agents are being introduced to clinical practice against other cancer selective signals [62].

Partial control of residual malignant cells forming metastatic lesions can be accomplished by metronomic treatment against regulatory $\mathrm{T}$ cells [63] and anti-angiogenic treatment that intends to minimize blood flow that seems essential for the highly metabolic active cancer cells [64].

Oncolytic viruses are also most promising future agents to eradicate malignant cells resistant to available anti-cancer modalities [65].

One promising future treatment of cancer including elimination of cancer stem cells may involve delivery of anti-cancer molecules such as tumor suppressor microRNAs or oncolytic viruses by mesenchymal stromal cells (MSCs) that naturally migrate to and target cancer cells [66].

The most recent modalities for improving anti-cancer immunotherapy by checkpoint inhibition of CTLA-4 by ipilimumab (Yervoy) [67] and PD-1 by nivolumab (Opdivo) or pembrolizumab (Keytruda) [68] or both combined [69] seem to further improve the therapeutic effects of anti-cancer immunotherapy. Finally, arming $\mathrm{T}$ lymphocytes with chimeric antigen receptors against cancer specific antigens (CAR-T) strategy may also develop into a most effective $\mathrm{T}$ cell dependent procedure for eradication of malignant cells including cells resistant to available chemotherapy [70].

In conclusion, based on the fact that despite major progress in medicine and the accumulative information about the biology and molecular constitution of cancer, much still remains unknown and cancer continues to represent one of the leading causes of death. Considering the fact that the last cancer cell, especially cancer stem cell, which is a priori resistant to available anticancer modalities is rarely eliminated, and failure of currently available medications to control rapidly progressive recurrent disease, cell-mediated immunotherapy may represent a safe and most effective treatment. That at a stage of MRD may result in cure.

\section{References}

1. Thomas ED (1983) Karnofsky Memorial Lecture. Marrow transplantation for malignant diseases. J Clin Oncol 1: 517-531.

2. Gratwohl A, Hermans J, Goldman JM, Arcese W, Carreras E, et al. (1998) Risk assessment for patients with chronic myeloid leukaemia before allogeneic blood or marrow transplantation. Chronic Leukemia Working Party of the European Group for Blood and Marrow Transplantation. Lancet 352: 1087-1092. 
3. Passweg JR, Rowlings PA, Armitage JO (1996) Report from the international bone /marrow transplant registry and autologous blood and marrow /transplant registry/North America. Clinical transplants, UCLA Tissue Typing Laboratory, Los Angeles, California.

4. Bast CR (1985) Principles of cancer biology: tumour immunology. In: DeVitta VT, Hellman S, Rosenberg SA, editors. Cancer; principles \& practice of oncology. J.B. Lippincott /Company, Philadelphia.

5. Weiss L, Lubin I, Factorowich Y, Lapidot Z, Reich S, et al. (1994) Effective graft vs leukemia effects independent of graft vs host disease after T-Cell depleted allogeneic bone marrow transplantation in a murine model of B Cell leukemia/lymphoma. Role of cell therapy and rIL-2. J Immunol 153: 2562-2567.

6. Grillo-López AJ, White CA, Dallaire BK, Varns CL, Shen CD, et al. (2000) Rituximab: the first monoclonal antibody approved for the treatment of lymphoma. Curr Pharm Biotechnol 1: 1-9.

7. Thomas ED (1963) The role of bone marrow transplantation in the eradication of malignant disease. Cancer 49:1963-1968.

8. Weiden PL, Sullivan KM, Fluornoy, Strob R, Thomas ED (1981) Antileukemic effect of chronic graft-versus-host disease: contribution to improved survival after allogeneic marrow transplantation. New Engl J Med 304: 1529-1533.

9. Weiden PL, Fluornoy N, Sanders JE, Sullivan KM, Thomas ED (1981) Anti-leukemic effect of graft-versus-host disease contributes to / improved survival after allogeneic marrow transplantation. Transplantation 13: 248-251.

10. Sullivan KM, Weiden PL, Storb R, Witherspoon RP, Fefer A, et al. (1989) Influence of acute and chronic graft-versus-host disease on relapse and survival after bone marrow transplantation from HLA-identical siblings as treatment of acute and chronic leukemia. Blood 73: 1720-1728.

11. Horowitz MM, Gale RP, Sondel PM, Goldman JM, Kersey J, et al. (1990) Graft-versus-leukemia reactions after bone marrow transplantation. Blood 75: 555-562.

12. Sinkovics JG, Shullenberger CC, Howe CD (1965) Prolongation and prevention of Rauscher virus mouse leukemia by spleen cells of naturally resistant or actively immunized mice. Clin Res 13: 36-39.

13. Boranic M, Tonkovic I (1971) Time pattern of the anti-leukemia effect of graft-versus-host reaction in mice, I. Cellular events. Cancer Res 31:1140-1147.

14. Bortin MM, Truitt RL, Rimm AA, Bach FH (1979) Graft-versusleukaemia reactivity induced by alloimmunisation without augmentation of graft-versus-host reactivity. Nature 281: 490-491.

15. Slavin S, Weiss L, Morecki S, Weigensberg M (1981) Eradication of murine leukemia with histoincompatible marrow grafts in mice conditioned with total lymphoid irradiation (TLI). Cancer Immunol Immunother 11:155-158.

16. Truitt RL, Shih CY, Lefever AV, Tempelis LD, Andreani M, et al. (1983) Characterization of alloimmunization-induced $\mathrm{T}$ lymphocytes reactive against AKR leukemia in vitro and correlation with graft-vs-leukemia activity in vivo. J Immunol 131: 2050-2058.

17. Meredith RF, O’Kunewick JP (1983) Possibility of graft-vs-leukemia determinants independent of the major histocompatibility complex in allogeneic marrow transplantation. Transplantation 35: 378-385.

18. Weiss L, Weigensberg M, Morecki S, Bar S, Cobbold S, et al. (1990) Characterization of effector cells of graft vs leukemia following allogeneic bone marrow transplantation in mice inoculated with murine B-cell leukemia. Cancer Immunol Immunother 31: 236-242.

19. Truitt RL, Atasoylu AA (1991) Impact of pre-transplant conditioning/and donor $\mathrm{T}$ cells on chimerism, graft-versus-host disease, graft-vs-leukemia reactivity, and tolerance after bone marrow transplantation. Blood 77: 2515-2523.

20. Moscovitch M, Slavin S (1984) Anti-tumor effects of allogeneic bone marrow transplantation in (NZB X NZW)F1 hybrids with spontaneous lymphosarcoma. J Immunol 132: 997-1000.

21. Morecki S, Moshel Y, Gelfend Y, Pugatsch T, Slavin S (1997) Induction of graft vs. tumor effect in a murine model of mammary adenocarcinoma. Int J Cancer 71: 59-63.
22. Morecki S, Yacovlev E, Diab A, Slavin S (1998) Allogeneic cell therapy for a murine mammary carcinoma. Cancer Res 58: 3891-3895.

23. Eibl B, Schwaighofer H, Nachbaur D, Marth C, Gächter A, et al. (1996) Evidence for a graft-versus-tumor effect in a patient treated with marrow ablative chemotherapy and allogeneic bone marrow transplantation for breast cancer. Blood 88: 1501-1508.

24. Ueno NT, Rondón G, Mirza NQ, Geisler DK, Anderlini P, et al. (1998) Allogeneic peripheral-blood progenitor-cell transplantation for poor-risk patients with metastatic breast cancer. J Clin Oncol 16: 986-993.

25. Or R, Ackerstein A, Nagler A, Kapelushnik J, Naparstek E, et al. (1998) Allogeneic cell-mediated immunotherapy for breast cancer after autologous stem cell transplantation: a clinical pilot study. Cytokines Cell Mol Ther 4: 1-6.

26. Higano CS, Brixey M, Bryant EM, Durnam DM, Doney K, et al. (1990) Durable complete remission of acute non-lymphocytic leukemia associated with discontinuation of immunosuppression following relapse after allogeneic bone marrow transplantation. A case report of a probable graft-versus-leukemia effect. Transplantation 50: 175-178.

27. Bacigalupo A, Van Lint MT, Occhini D, Gualandi F, Lamparelli T, et al. Increased risk of leukemia relapse with high dose cyclosporine A after allogeneic marrow transplantation for acute leukemia. Blood 77: 1423-1428.

28. Weiss L, Reich S, Slavin S (1990) Effect of cyclosporine A and methylprednisolone on the GVL effect across major histocompatibility barriers in mice following allogeneic bone marrow transplantation. Bone Marrow Transplant 6: 229-233.

29. Kapelushnik J, Or R, Aker M (1996) Allogeneic cell therapy of severe beta thalassemia major by displacement of host stem cells in mixed chimera by donor blood lymphocytes. Bone Marrow Transplant 19: 96-98.

30. Kapelushnik J, Aker M, Or R (1997) Allogeneic cell therapy as a new modality for displacement of genetically abnormal stem cells as part of the conditioning for allogeneic bone marrow transplantation. Correction of genetic diseases by transplantation (Chapter 4). Cogent Press, Washington, USA.

31. Aker M, Kapelushnik J, Pugatsch T, Naparstek E, Ben-Neria S, et al. (1998) Donor lymphocyte infusions to displace residual host hematopoietic cells after allogeneic BMT for beta thalassemia major. J Pediatr Hematol/Oncol 20: 145-148.

32. Slavin S, Nagler A, Naparstek E (1999) Mini-transplants and cell//based therapies for malignant and non-malignant disorders. Curr Opin Organ Transplant 4: 184-188.

33. Slavin S, Or R, Naparstek E, Ackerstein A, Weiss L (1988) Cellularmediated immunotherapy of leukemia in conjunction with autologous and allogeneic bone marrow transplantation in experimental animals and man. Blood 72: 407a.

34. Slavin S, Naparstek E, Nagler A, Ackerstein A, Kapelushnik J, et al. (1995) Allogeneic cell therapy for relapsed leukemia after bone marrow transplantation with donor peripheral blood lymphocytes. Exp Hematol 23: 1553-1562.

35. Slavin S, Naparstek E, Nagler A, Ackerstein A, Samuel S, et al. (1996) Allogeneic cell therapy/with donor peripheral blood cells and recombinant human interleukin-2 to treat leukemia relapse post allogeneic bone marrow transplantation. Blood 87: 2195-2204.

36. Kolb HJ, Mittermüller J, Clemm C, Holler E, Ledderose G, et al. (1990) Donor leukocyte transfusions for treatment of recurrent chronic myelogenous leukemia in marrow transplant patients. Blood 76: 2462-2465.

37. Kolb HJ, Schattenberg A, Goldman JM, Hertenstein B, Jacobsen N, et al. (1995) Graft-versus-leukemia effect of donor lymphocyte transfusions in marrow grafted patients: European Group for blood and marrow transplantation. Blood 86: 2041-2050.

38. Collins RH Jr, Shpilberg O, Drobyski WR, Porter DL, Giralt S, et al. (1997) Donor leukocyte infusions in 140 patients with relapsed malignancy after allogeneic bone marrow transplantation. J Clin Oncol 15: 433-444. 
39. Porter DL, Roth MS, McGarigle C, Ferrara JL, Antin JH (1994) Induction of graft-versus-host disease as immunotherapy for relapsed chronic myeloid leukemia. N Engl J Med 330: 100-106.

40. Mackinnon S, Papadopoulos EB, Carabassi MH, Reich L, Collins NH, et al. (1995) Adoptive immunotherapy evaluating escalating doses of donor leukocytes for relapse of chronic myeloid leukemia after bone marrow transplantation: separation of graft-versus-leukemia responses from graft-versus-host disease. Blood 86: 1261-1268.

41. Slavin S, Morecki S, Weiss L, Or R (2003) Immunotherapy of hematologic malignancies and metastatic solid tumors in experimental animals and man. Crit Rev Oncol Hematol 46: 139-163.

42. Naparstek E, Or R, Nagler A, Cividalli G, Engelhard D, et al. (1995) Tcell-depleted allogeneic bone marrow transplantation for acute leukaemia using Campath-1 antibodies and post-transplant administration of donor's peripheral blood lymphocytes for prevention of relapse. Brit J Haematol 89: 506-515.

43. Giralt S, Estey E, Albitar M, van Besien K, Rondon G, et al. (1997) Engraftment of allogeneic hematopoietic progenitor cells with purine analog-containing chemotherapy: Harnessing graft-vs-leukemia without myeloablative therapy. Blood 89: 4531-4536.

44. Slavin S, Nagler A, Naparstek E, Kapelushnik J, Aker M, et al. (1998) Non-myeloablative stem cell transplantation and cell therapy as an alternative to conventional bone marrow transplantation with lethal cytoreduction for the treatment of malignant and non malignant hematologic diseases. Blood 91: 756-763.

45. Childs R, Chernoff A, Contentin N, Bahceci E, Schrump D, et al. (2000) Regression of metastatic renal-cell carcinoma after nonmyeloablative allogeneic peripheral-blood stem-cell transplantation. N Engl J Med 343: 750-758.

46. Slavin S (2000) Immunotherapy of cancer with alloreactive lymphocytes. N Engl J Med 343: 802-803.

47. Slavin S (2001) Immunotherapy of cancer with alloreactive lymphocytes. Lancet Oncol 2: 491-498.

48. Slavin S (2005) Allogeneic cell-mediated immunotherapy at the stage of minimal residual disease following high-dose chemotherapy supported by autologous stem cell transplantation. Acta Haematol 114: 214-220.

49. Or R, Ackerstein A, Nagler A, Amar A, Naparstek E, et al. (1998) Allogeneic cell-activated immunotherapy for malignant lymphoma at the stage of minimal residual disease after stem cell transplantation. J Immunother 21: 447-453.

50. Slavin S, Ackerstein A, Or R, Shapira MY, Gesundheit B, et al. (2010) Immunotherapy in high-risk chemotherapy-resistant patients with metastatic solid tumors and hematological malignancies using intentionally mismatched donor lymphocytes activated with rIL-2: a phase I study. Cancer Immunol Immunother 59: 1511-1519.

51. Ruggeri L, Mancusi A, Capanni M, Urbani E, Carotti A, et al. (2007) Donor natural killer cell allorecognition of missing self in haploidentical hematopoietic transplantation for acute myeloid leukemia: challenging its predictive value. Blood 110: 433-440.

52. Velardi A, Ruggeri L, Mancusi A, Aversa F, Christiansen FT (2009) Natural killer cell allorecognition of missing self in allogeneic hematopoietic transplantation: a tool for immunotherapy of leukemia. Curr Opin Immunol 21: 525-530.

53. Morecki S, Lindhofer H, Yacovlev E, Gelfand Y, Slavin S (2006) Use of trifunctional bispecific antibodies to prevent graft versus host disease induced by allogeneic lymphocytes. Blood 107: 1564-1569.

54. Morecki S, Lindhofer H, Yacovlev E, Gelfand Y, Ruf P, et al. (2008) Induction of long-lasting antitumor immunity by concomitant cell therapy with allogeneic lymphocytes and trifunctional bispecific antibody. Exp Hematol 36: 997-1003.

55. Eckerstein A, Slavin S, Weiss L, Naparstek E (1990) Immunotherapy in conjunction with autologous bone marrow transplantation. Bone Marrow Transplant 5 Suppl 1: 38.

56. Nagler A, Ackerstein A, Or R, Naparstek E, Slavin S (1997) Immunotherapy with recombinant human interleukin-2 and recombinant interferon-alpha in lymphoma patients postautologous marrow or stem cell transplantation. Blood 89: 3951-3959.

57. Slavin S, Nagler A (1998) Immunotherapy in conjunction with autologous and allogeneic blood or marrow transplantation in lymphoma. Ann Oncol 9 Suppl 1: S31-39.

58. Nagler A, Berger R, Ackerstein A, Czyz JA, Diez-Martin JL, et al. (2010) A randomized controlled multicenter study comparing recombinant interleukin 2 (rIL-2) in conjunction with recombinant interferon alpha (IFN-alpha) versus no immunotherapy for patients with malignant lymphoma post autologous stem cell transplantation. J Immunother 33: 326-333.

59. Yamamoto N, Naraparaju VR (1998) Structurally well-defined macrophage activating factor derived from vitamin D3-binding protein has a potent adjuvant activity for immunization. Immunol Cell Biol 76: 237-244.

60. Pacini S, Punzi T, Morucci G, Gulisano M, Ruggiero M (2012) Effects of vitamin D-binding protein-derived macrophage-activating factor on human breast cancer cells. Anticancer Res 32: 45-52.

61. Druker BJ, Talpaz M, Resta DJ, Peng B, Buchdunger E, et al. (2001) Efficacy and safety of a specific inhibitor of the BCR-ABL tyrosine kinase in chronic myeloid leukemia. N Engl J Med 344: 1031-1037.

62. Carmi C, Mor M, Petronini PG, Alfieri RR (2012) Clinical perspectives for irreversible tyrosine kinase inhibitors in cancer. Biochem Pharmacol 84: 1388-1399.

63. Ye J, Livergood RS, Peng G (2013) The role and regulation of human Th17 cells in tumor immunity. Am J Pathol 182: 10-20.

64. Young RJ, Reed MW (2012) Anti-angiogenic therapy: concept to clinic. Microcirculation 19: 115-125.

65. Alemany R (2013) Viruses in cancer treatment. Clin Transl Oncol 15: 182-188.

66. Lee HK, Finniss S, Cazacu S, Bucris E, Ziv-Av A, et al. (2013) Mesenchymal stem cells deliver synthetic microRNA mimics to glioma cells and glioma stem cells and inhibit their cell migration and selfrenewal. Oncotarget 4: 346-361.

67. Eggermont AM, Chiarion-Sileni V, Grob JJ, Dummer R, Wolchok JD, et al. (2015) Adjuvant ipilimumab versus placebo after complete resection of high-risk stage III melanoma (EORTC 18071): a randomised, doubleblind, phase 3 trial. Lancet Oncol 16: 522-530.

68. Weber JS, D'Angelo SP, Minor D, Hodi FS, Gutzmer R, et al. (2015) Nivolumab versus chemotherapy in patients with advanced melanoma who progressed after anti-CTLA-4 treatment (CheckMate 037): a randomised, controlled, open-label, phase 3 trial. Lancet Oncol 16: 375-384.

69. Postow MA, Chesney J, Pavlick AC, Robert C, Grossmann K, et al. (2015) Nivolumab and Ipilimumab versus Ipilimumab in Untreated Melanoma. N Engl J Med .

70. Eshhar Z (2014) From the mouse cage to human therapy: a personal perspective of the emergence of $\mathrm{T}$-bodies/chimeric antigen receptor $\mathrm{T}$ cells. Hum Gene Ther 25: 773-778. 